\title{
Desarrollo y análisis de un sistema para la determinación de la dinámica del movimiento más general de la azotea de un edificio de gran altura y su evolución en el tiempo
}

Septiembre de 2015

Nieves Quesada Olmo 


\section{Índice general}

Resumen III

Índice general $\quad$ IX

1 Introducción 1

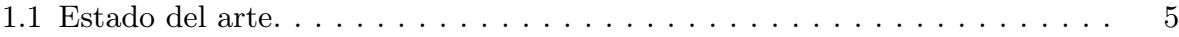

1.2 Marco arquitectónico. . . . . . . . . . . . . . . . . . . 11

1.2.1 Por qué construimos . . . . . . . . . . . . . . . . . . . . . . . . . . . . 11

$1.2 .2 \mathrm{El}$ síndrome del rascacielos . . . . . . . . . . . . . . . . . . . 17

1.3 Estructura formal de la Tesis . . . . . . . . . . . . . . . . . . . . . 29

2 Método de los Ajustes Coordinados 33

2.1 Caso General . . . . . . . . . . . . . . . . . . . . . . . . . . . . 35

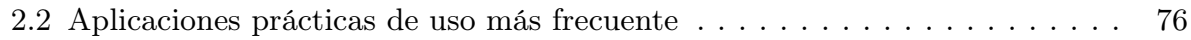

2.2.1 Adición de variables o parámetros que también presentan la condición de

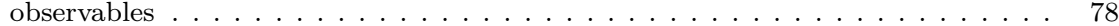

2.2 .2 Adición de funciones de variables o parámetros. . . . . . . . . . . . . . . . 93

2.2 .3 Supresión de observables . . . . . . . . . . . . . . . . . . . . . . . 103

3 El control microgeodésico de deformaciones. Definición teórica del umbral de precisión o sensibilidad

3.1 Antecedentes . . . . . . . . . . . . . . . . . . . . . . 105

3.2 Desarrollo general. . . . . . . . . . . . . . . . . . . . . . . . . . . . 108 
3.3 Interpretación estadística de resultados . . . . . . . . . . . . . . . . . . 115

3.3.1 Formulación básica a partir del método general de Ajustes Coordinados . . 116

3.3.2 Test estadísticos de control . . . . . . . . . . . . . . . . . . . . . . . . 124

3.4 Test Específico de control Microgeodésico de deformaciones J/Q-Test . . . . 135

3.4.1 Sobre la sensibilidad de redes . . . . . . . . . . . . . . . . . . . . . . 141

3.4.2 Estimación de la sensibilidad teórica a partir del W-Test de Baarda . . . . . 143

3.4.3 Una cuestión complementaria: la sensibilidad específica . . . . . . . . . 145

4 Aplicación práctica para la determinación del umbral de precisión o sensibilidad

4.1 Introducción: el J/Q Test. . . . . . . . . . . . . . . . . . . . . . . . . . . . . 151

4.2 Definición del sistema de formas lineales . . . . . . . . . . . . . . . . . 153

4.3 Sensibilidad PRÁCTICA . . . . . . . . . . . . . . . . . . . 160

4.4 Sensibilidad TEÓRICA según Baarda . . . . . . . . . . . . . . . . . . 164

4.4 .1 Fiabilidad interna de la red . . . . . . . . . . . . . . . . . 164

4.4.2 Fiabilidad externa de la red: comprobación de los observables . . . . . . . 166

4.5 Valores más probables de sensibilidad TEÓRICA Y PRÁCTICA Red de Prueba UPV . . . . . . . . . . . . . . . . . . . . . . . . . . . . . . . . . . 170

4.5.1 Sensibilidad PRÁCTICA más probable . . . . . . . . . . . . . . 170

4.5.2 Sensibilidad TEÓRICA más probable . . . . . . . . . . . . . . . 174

5 Propuesta metodológica para analizar la dinámica más general: ensayos experimentales preliminares 177

5.1 Introducción . . . . . . . . . . . . . . . . . . . . . . . . . . 177

5.2 El modelo matemático de ajuste y su resolución general . . . . . . . . . . 178

5.2.1 Implementación del Método de Incrementos de Coordenadas al control de deformaciones. . . . . . . . . . . . . . . . . . . . . 181

5.2.2 Matriz de diseño $A_{1}$, matriz de $A_{2}$ de condiciones y vectores de términos independientes $K_{1}$ y $K_{2} \ldots \ldots \ldots \ldots \ldots \ldots \ldots \ldots$

5.3 Resolución del ajuste . . . . . . . . . . . . . . . . . . . . . . . . . . . 189

5.3.1 Solución determinista con variable $d$ y adición de ecuaciones de condición . 193 5.3.2 Solución determinista con variable $d$ sin ecuaciones de condición . . . . . . . 196

5.4 Solución de red libre . . . . . . . . . . . . . . . . . . . . . . . . . . . . . 196 
6 Aplicación a un edificio de gran altura: Torre Espacio

6.1 La Torre . . . . . . . . . . . . . . . . . . . . . . . . . . . . . . 199

6.2 Planteamiento básico: físico, matemático y probabilístico . . . . . . . . . . . 221

6.3 Preparando la base de observación. . . . . . . . . . . . . . . . . 227

6.4 Valores más probables de sensibilidad PRÁCTICA Y TEÓRICA en Torre

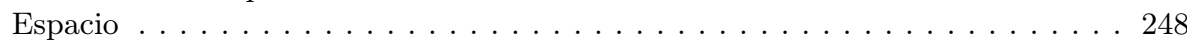

6.4.1 Aplicación del F-Test de Fisher-Snedecor Generalizado. . . . . . . . . . . . 255

6.4.2 Comprobación del umbral de sensibilidad definido. . . . . . . . . . . . . . 259

6.4.3 Sobre el umbral de sensibilidad y el filtrado de observables . . . . . . . . . 261

6.4 .4 Resultados y conclusiones. . . . . . . . . . . . . . . . . . 275

6.5 Validación de la metodología en Torre Espacio . . . . . . . . . . . . . . 277

7 Conclusiones y futuras líneas de investigación 295

$\begin{array}{ll}\text { Bibliografía } & 299\end{array}$ 


\section{Índice de figuras}

1.1. Maqueta de distintas perspectivas del "Shanghai World Financial Center" .................... . . 7

1.2. Iglesia de la Colonia Güell de A. Gaudí . . . . . . . . . . . . . . . 13

1.3. Azotea de la Casa Milà de A. Gaudí . . . . . . . . . . . . . . . . . 14

1.4. Auditorio y Palacio de Congresos de Castellón de C. Ferrater . . . 15

1.5. Estatua de La Libertad y horizonte de Manhattan desde la Isla de Ellis . . . . . . . . . . . . . . . . . 18

1.6. Vista de las Torres Gemelas desde la plaza del World Trade Center 19

1.7. Vista de la City londinense desde la otra orilla del Támesis . . . . 20

1.8. "Milleniun Tower" a orillas del Danubio en Viena . . . . . . . . . . 21

1.9. Hotel de siete estrellas Burj Al Arab "La Torre de los Árabes" en

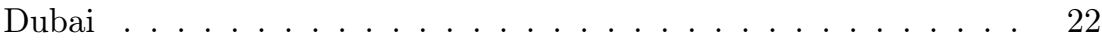

1.10. "Dongbu Financial Center" en Corea del Sur . . . . . . . . . . . . . 23

1.11. Edificio residencial "Highcliff" en la bahía de Hong Kong . . . . . . 25

1.12. "Taipei 101" en Taiwan . . . . . . . . . . . . . . . . . . 26

1.13. Panorámica del distrito financiero de Pudong en Shanghai . . . . . 27

1.14. "Burj Khalifa" en Dubai . . . . . . . . . . . . . . . . . . . 28

3.1. Ejemplo de distribución $\chi_{8}^{2}$ y $\chi_{8,20}^{\prime 2} \ldots \ldots$. . . . . . . . . . . . 137

3.2. Distribución $\chi_{n, \lambda}^{\prime 2}$ con diversos parámetros de traslación $\lambda$. . . . . 139 
4.1. Curvas de distribución $\chi_{8}^{2}$ y $\chi_{8,20}^{\prime 2} \ldots \ldots$. . . . . . . . . . . 154

4.2. Diversas curvas de distribución $\chi_{n}^{2}$ y $\chi_{n, \lambda}^{\prime 2} \ldots \ldots$. . . . . . . . . 161

5.1. Localización de la Red de Prueba formada por los vértices $V 1, V 2$, $V 3$ y $V 4 \ldots \ldots \ldots \ldots \ldots . \ldots \ldots \ldots$

5.2. Croquis de la red . . . . . . . . . . . . . . . . . . 179

5.3. Posible desplazamiento del vértice $V 4$. . . . . . . . . . . . . . . 195

5.4. Posible desplazamiento del vértice $V 1$. . . . . . . . . . . . . . 195

6.1. Torre Espacio . . . . . . . . . . . . . . . . . 200

6.2. Torre Espacio: fachada Este . . . . . . . . . . . . . . . . . 202

6.3. Planta 6, de base totalmente cuadrada . . . . . . . . . . . . . 203

6.4. Planta 26, evolución según la curva coseno desplazada . . . . . . . 204

6.5. Planta 33, evolución según la curva coseno desplazada . . . . . . . 205

6.6. Planta 52, intersección de dos arcos de circunferencia de $90^{\circ}$. . . 206

6.7. Definición del diseño en planta de Torre Espacio . . . . . . . . . . 207

6.8. Definición diseño fachadas Sur y Este de Torre Espacio . . . . . . . 208

6.9. Distribución de los ascensores en el núcleo principal de comunicaciones 211

6.10. Sistema de climatización por "aire frío" . . . . . . . . . . . . 217

6.11. Cuadrilátero testigo indeformable V1V2V3V4 . . . . . . . . . . . 222

6.12. Situación de los vértices $V_{1} V_{2} V_{3} V_{4}$ en la planta coronación de Torre Espacio .......................... 223

6.13. Esquema representativo del desplazamiento plano de la azotea . . . 224

6.14. Dos posiciones genéricas distintas del vértice V3 . . . . . . . . 226

6.15. Panorámica de la zona central diáfana cubierta por el entramado metálico en la azotea . . . . . . . . . . . . . . . . . . 229

6.16. Parte útil de la azotea ocupada por el carril de la góndola de limpieza de fachadas . . . . . . . . . . . . . . . . 230 
6.17. Disposición definitiva de las antenas GNSS en Torre Espacio . . . . 231

6.18. Detalle de la base nivelante que permite el estacionamiento en los pilares de la azotea de Torre Espacio . . . . . . . . . . . . . . . 231

6.19. Observación con Estación Total robotizada sobre el vértice 2 en Torre Espacio . . . . . . . . . . . . . . . . . . 232

6.20. Observación clásica, situación del prisma en el vértice 4 . . . . . . 233

6.21. Antena GNSS 1 sobre pilar metálico en la azotea de Torre Espacio 234

6.22. Antena GNSS 2 sobre pilar metálico en la azotea de Torre Espacio 235

6.23. Antena GNSS 3 sobre pilar metálico en la azotea de Torre Espacio 235

6.24. Antena GNSS 4 sobre pilar metálico en la azotea de Torre Espacio 236

6.25. Detalle de los 4 receptores GM10 de Leica situados en la planta 55 de Torre Espacio . . . . . . . . . . . . . . . 237

6.26. Sala técnica que alberga el "rack de comunicaciones" en la planta 55 de Torre Espacio . . . . . . . . . . . . . . . . . 238

6.27. Menú "View" del software Leica GNSS Spider . . . . . . . . . . . . 240

6.28. Menú "Tools" del software Leica GNSS Spider . . . . . . . . . . . . 241

6.29. Opción Edit Site del software Leica GNSS Spider . . . . . . . . . . 242

6.30. Verificación de recepción de datos con Software Leica GNSS Spider 243

6.31. Configuración de ficheros Rinex, "File Products", con software Leica GNSS Spider ... . . . . . . . . . . . . . 244

6.32. Configuración del posicionamiento en Tiempo Real, "RT Positioning", con software Leica GNSS Spider . . . . . . . . . . . . 245

6.33. Configuración del posicionamiento en postproceso, "PP Positioning", con software Leica GNSS Spider . . . . . . . . . . . . . . . . . . 246

6.34. Inicialización de cálculo con el software Leica GNSS Spider . 247

6.35. Antena GNSS 1 sobre pilar metálico en la azotea de Torre Espacio 249

6.36. Antena GNSS 2 sobre pilar metálico en la azotea de Torre Espacio 249

6.37. Curvas $F$ y $F^{\prime}$ de Snedecor con $\alpha=8,085 \cdot 10^{-5}$ y $\beta=0,9542 \quad$. 257

6.38. Curvas $F$ y $F^{\prime}$ de Snedecor con $\alpha=0,0045$ y $\beta=0,8929$. . . . 258 
6.39. Vista de la planta de coronación de Torre Espacio con la posición del cuadrilátero formado por las antenas . . . . . . . . . . . 278

6.40. Localización vértice fijo IGNE y vértices libres de Torre Espacio 279

6.41. Condicionado de obligado cumplimiento de Torre Espacio . . . . . 281

6.42. Probabilidad simultánea de las 4 antenas GNSS . . . . . . . . . . . 284

6.43. Muestra de la precisión instantánea de las antenas GNSS . . . . . 285

6.44. Desplazamiento en tiempo real, precisión instantánea y desplazamiento de los últimos 5 minutos de la antena $1 \ldots$. . . . . . . 286

6.45. Desplazamiento en tiempo real, precisión instantánea y desplazamiento de los últimos 5 minutos de la antena $2 \ldots$. . . . . . 287

6.46. Desplazamiento en tiempo real, precisión instantánea y desplazamiento de los últimos 5 minutos de la antena 4 . . . . . . . . . 288

6.47. Sistema de alerta mediante SMS desarrollado en la App . . . . . . 289

6.48. Gráfica mensual de envíos de SMS . . . . . . . . . . . . . . . . . . 290

6.49. Mástil de la bandera junto al vértice 2 . . . . . . . . . . . . . 291

6.50. Día 23 de Febrero de 2015: desplazamiento de los últimos 5 minutos de las antenas 1 y $2 \ldots \ldots . \ldots . \ldots . \ldots . . \ldots 293$

6.51. Día 23 de Febrero de 2015: desplazamiento de los últimos 5 minutos de las antenas 3 y 4 . . . . . . . . . . . . . . . 294 


\section{Índice de tablas}

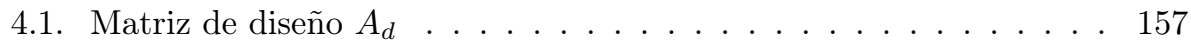

4.2. Vector de términos independientes $K_{d} \ldots \ldots \ldots$. . . . . . 158

4.3. Coordenadas cartesianas geocéntricas del vértice fijo Valencia2 159

4.4. Fiabilidad externa de la red . . . . . . . . . . . . . 167

4.5. Sensibilidades teóricas $\lambda_{T}$ y prácticas $\lambda_{P}$ de los momentos 1-8, 1-5 y $1-6 \ldots \ldots \ldots \ldots 17 \ldots \ldots \ldots$

4.6. Vector $d_{P}$, variable estadística $\lambda_{P}$, nivel de significación $\alpha$ y potencia del test $\beta \ldots \ldots \ldots$. . . . . . . . . . . . . . 171

4.7. Vector $d_{T}$, variable estadística $\lambda_{T}$, nivel de significación $\alpha$ y potencia del test $\beta \ldots \ldots \ldots \ldots$. . . . . . . . . . . . 174

5.1. Diferencias entre las distancias GNSS y distanciometricas . . . . . 189

5.2. Incrementos de coordenadas Valencia2 - vértice V1 . . . . . . . . . 192

5.3. Incrementos de coordenadas entre los vértices libres de la red de prueba .......................... 193

5.4. Desplazamientos de los vértices del cuadrilátero de la primera solución determinista . . . . . . . . . . . . . . . . . . . . . 194

5.5. Correcciones $x_{1}$ y $x_{2}$ de los vértices del cuadrilátero de la primera solución determinista . . . . . . . . . . . . . . . . . . 194

5.6. Desplazamientos $d_{x^{\prime}}, d_{y^{\prime}}$ de los vértices del cuadrilátero de la primera solución determinista 
5.7. Desplazamientos de los vértices del cuadrilátero de la segunda solución determinista . . . . . . . . . . . . . . . 196

5.8. Desplazamientos de los vértices del cuadrilátero de la solución libre 197

6.1. Parámetros que definen la sensibilidad práctica con momentos clase $\mathrm{A}, \mathrm{B}, \mathrm{C}, \mathrm{D} \mathrm{y} \mathrm{E} \mathrm{...} \mathrm{.} \mathrm{.} \mathrm{.} \mathrm{.} \mathrm{.} \mathrm{.} \mathrm{.} \mathrm{.} \mathrm{.} \mathrm{.} \mathrm{.} \mathrm{.} 251$

6.2. Parámetros que definen la sensibilidad teórica con momentos clase $\mathrm{A}, \mathrm{B}, \mathrm{C}, \mathrm{D}$ y E . . . . . . . . . . . . . . . . 252

6.3. Parámetros de sensibilidad práctica y teórica del ajuste con momento de clase C . . . . . . . . . . . . . . . . . . . . 254

6.4. Cálculo del círculo de error y valor más probable de sensibilidad práctica con momentos clase C y D . . . . . . . . . . . 255

6.5. Parámetros de sensibilidad práctica con momento de clase C . . . 260

6.6. Cálculo del vector deformación $d_{m p}$ con momentos de clase C, C'y

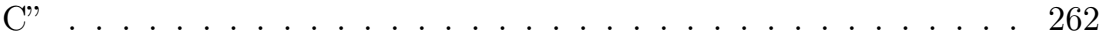

6.7. Parámetros de sensibilidad práctica con momentos de clase C, C'y

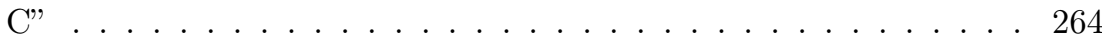

6.8. Parámetros de sensibilidad teórica con momentos de clase C, C' y C' 265

6.9. Cálculo del vector deformación $d_{m p}$ con momentos de clase D, D'y D" . . . . . . . . . . . . . . . . . . 267

6.10. Parámetros de sensibilidad práctica con momentos de clase D, D' y

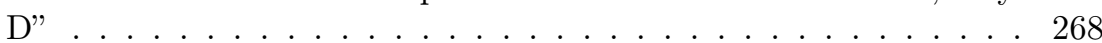

6.11. Parámetros de sensibilidad teórica con momentos de clase D, D’ y D”269

6.12. Cálculo del vector deformación $d_{m p}$ con momentos de clase C, $C_{s f}$,

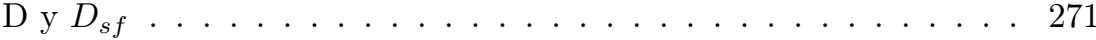

6.13. Parámetros de sensibilidad práctica con momentos de clase $\mathrm{C}, C_{s f}$, $\mathrm{D}$ y $D_{s f} \ldots \ldots \ldots \ldots \ldots \ldots \ldots$. . . . . . . . . . . . . . . . . . . . . . .

6.14. Parámetros de sensibilidad teórica con momentos de clase C, $C_{s f}$, $\mathrm{D}_{\mathrm{y}} D_{s f} \ldots \ldots \ldots \ldots \ldots \ldots \ldots \ldots \ldots \ldots$

6.15. Cálculo del círculo de error y valor más probable de sensibilidad práctica del momento de clase C . . . . . . . . . . . . 275 
6.16. Parámetros de sensibilidad práctica y teórica y valores del nivel de significación $\alpha$ y potencia del test $\beta$ del ajuste con momento de clase C276 